\title{
Identifying the Multiple Contexts of a Situation
}

\author{
Aviv Segev \\ Technion - Israel Institute of Technology \\ Haifa 32000 \\ Israel \\ asegev@technion.ac.il
}

\begin{abstract}
The paper presents a contexts recognition algorithm that uses the Internet as a knowledge base to extract the multiple contexts of a given situation, based on the streaming in text format of information representing the situation. Context is represented here as any descriptor most commonly selected by a set of subjects to describe a given situation. Multiple contexts are matched with the situation. The algorithm yields consistently good results and the comparison of the algorithm results with the results of people showed that there was no significant difference in the determination of context.

Keywords: Matching context, Context recognition, Metadata, Text analysis
\end{abstract}

\section{Introduction}

The question of context recognition is defined as one of the main questions addressed by the international interdisciplinary context community [1]. A context is a descriptor (such as a word or an image) or a set of descriptors that defines a situation. A context can convey a different facet, a different point of view, or a different understanding of a situation. Therefore, many situations are characterized by several different contexts. This paper presents an algorithm of context recognition that analyzes a given situation, represented in text format, and identifies its multiple contexts, textual descriptors. The performance of the algorithm was compared to the human process of multiple contexts recognition and yielded consistently good results.

Section 2 reviews related works in the literature. Section 3 presents a formal definition of contexts recognition and divides the problem into two sub-problems. Section 4 describes the contexts recognition algorithm, which consists of five main processes: the collection of data, the selection of contexts for each text, the ranking of the contexts, the identification of the current contexts, and the union of synonymous contexts, and describes the use of the Internet as a context database. Section 5 presents the analysis of the algorithm. Finally, section 6 presents some conclusions and directions of future research.

\section{Related Work}

\subsection{Formal Definition of Context}

Context is defined as a descriptor (such as a word or an image) or set of descriptors that can represent a situation or a scenario. A scenario is defined as "the 
world state", a situation that is a snapshot or an instance of the world at some given time, namely, all attributes of the world, including all objects, their properties and internal states, and the relationships between them [11].

McCarthy [9] defined the formalization of the notion of context as one of the main problems in the field of artificial intelligence (AI) and argued that a most general context does not exist. Consequently, McCarthy [10] worked to formalize context and to develop a theory of introducing context as formal objects.

Since a situation is characterized by many different features, it may have multiple contexts. McCarthy's formal definition of context is used in this work to identify the multiple contexts of a situation.

\subsection{Blackboard Model}

The model architecture for the contexts recognition is based on the blackboard model [3]. The blackboard model has been used in many AI applications, e.g., understanding images, signals, and speech, to represent possible solutions to a given problem. Blackboard is used here to enhance information extraction from more than one information source. For example, different information sources can be multiple people having multiple conversations at the same place and time, as in the case of Internet chats.

\subsection{Information Seeking}

Information seeking is the process in which people turn to information resources to increase their level of knowledge regarding their goals [4]. Although the basic concept of information seeking remains unchanged, the growing need for the automation of the process has called for innovative tools to assign some of the tasks involved in information seeking to the machine level. Thus, techniques for information seeking based on textual information are used, including the ontology tools Text-To-Onto [8], OntoMiner [6], and TexaMiner [7], to name a few, and databases are extensively used for the efficient storage and retrieval of information.

The Internet can be seen as a large database that is constantly being modified and updated. Many information seeking techniques have been developed to retrieve information from the Internet. For example, Valdes-Perez and Pereira [13] developed an algorithm based on the concise all pairs profiling (CAPP) clustering method. This method approximates profiling of large classifications. Use of hierarchical structure was explored for classifying a large, heterogeneous collection of web content [2]. Another method involves checking the frequency of the possible keyphrases of articles using the Internet [12]. However, this method is based on an existing set of keywords and uses the Internet for ranking purposes only.

The present algorithm attempts to automate context recognition, based on information seeking techniques, using the Internet as a database for possible multiple contexts. The algorithm differs from previous text analysis techniques by allowing the input to be received from multiple sources, in an unstructured format. In addition, the algorithm utilizes data resources that are independent of the user and are constantly changing to analyze the information. 


\section{Formal Definition of the Problem}

McCarthy [10] formalized context as first class objects with the following basic relation:

ist $(\mathcal{C}, \mathrm{P})$ meaning that the proposition $\mathrm{P}$ is true in the context $\mathcal{C}$.

In this paper context is defined as any textual description that is most commonly selected by a set of subjects to describe a given situation:

Let $P_{1}, P_{2}, \ldots, P_{m}$ be a series of textual propositions defining situation $\mathrm{S}$.

Contexts $C_{1}, C_{2}, \ldots, C_{k}$ are defined as the contexts of situation $\mathrm{S}$ if:

$\exists n$ subjects, $n \geqslant 1$ so for the majority of n selected

$\operatorname{ist}\left(\mathcal{C}_{i}, P_{j}\right) \forall \mathrm{i}$, for a given $\mathrm{j}$

(Contexts $C_{1}, C_{2}, \ldots, C_{k}$ are true for textual proposition $P_{j}$ )

For a series of propositions there exists a collection of sets of contexts.

Let $P_{1}, P_{2}, \ldots, P_{m}$ be a series of textual propositions when $\forall P_{i}$ there exists a collection of sets of contexts $C_{i j}$ so that:

$\forall \mathrm{i}$, ist $\left(\mathcal{C}_{i j}, P_{i}\right) \forall \mathrm{j}$ meaning that the textual proposition $P_{i}$ is true in each of the set of contexts $\mathcal{C}_{i j} . \mathcal{C}_{i j}$ are not predefined hierarchically in a structure such as a tree. However, hierarchical structures can be built according to a specific set of textual propositions.

The main research problem is formally defined as:

What is the outer context $\mathcal{C}$ defined by

$i s t\left(\mathcal{C}, \bigcap_{i=1}^{m} i s t\left(\mathcal{C}_{i j}, P_{i}\right)\right) \forall j$.

The number of existing contexts is assumed to be finite and to satisfy

$\mathcal{C}, \mathcal{C}_{i j} \subseteq U_{c}$ (unity of all existing contexts)

The main research problem can be divided into two sub-problems:

1. Let $\mathrm{P}$ be a given single text. What are the possible contexts $\mathcal{C}_{i}$ that satisfy $\operatorname{ist}\left(\mathcal{C}_{i}, \mathrm{P}\right) \forall \mathrm{i}$ (for single text $\mathrm{P}$ all contexts $\mathcal{C}_{i}$ are true)

2. Let $P_{1}, P_{2}, \ldots, P_{m}$ be a set of texts that satisfy the following condition: for each text $P_{i}$ there exists a set of contexts $\mathcal{C}_{i j}$ so that ist $\left(\mathcal{C}_{i j}, P_{i}\right) \forall \mathrm{j}$.

What is the outer context $\mathcal{C}$ so that $i s t\left(\mathcal{C}, \bigcap_{i=1}^{m} i s t\left(\mathcal{C}_{i j}, P_{i}\right)\right) \forall j$.

The division of the problem into two parts allows a solution of the first part to be acquired by information seeking through the Internet. The second part is addressed using an algorithm that ranks the contexts according to the importance of the information retrieved in the first part. The result of the following algorithm is a list of contexts, which forms the outer context of the situation.

\section{The Contexts Recognition Algorithm}

The research algorithm is based on the streaming in text format of information that represents input from different sources, such as Internet chats. The contexts recognition algorithm output is a set of contexts that attempt to describe the current situation most accurately. The set of contexts is a list of words or phrases, each describing an aspect of the situation. Thus, multiple contexts can be matched to a given situation. The algorithm, which is outlined in Figure 1, consists of five major processes:

- Collecting Data - The information from the information sources is decomposed into words and the keywords are extracted from them. 


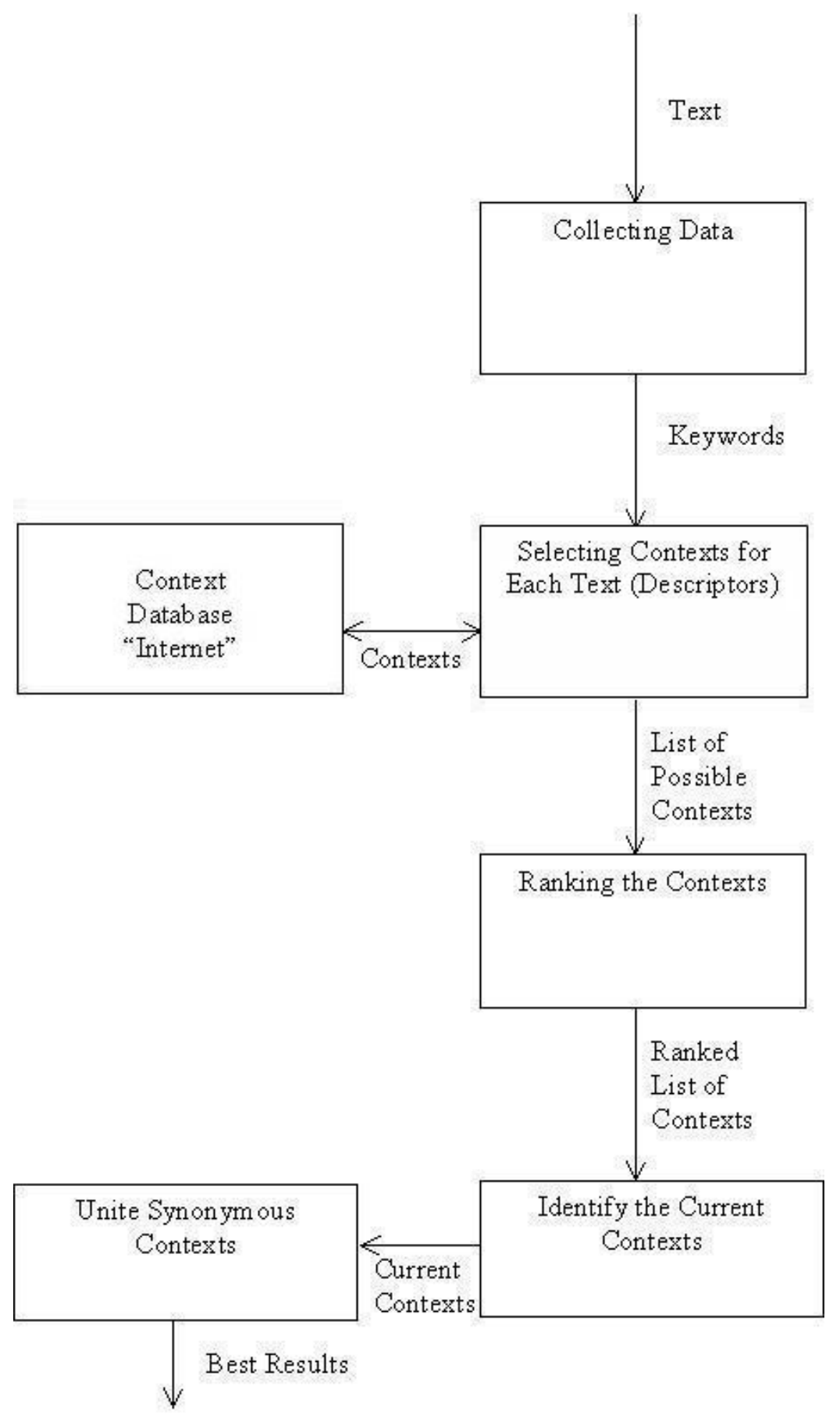

Fig. 1. The Contexts Recognition Algorithm Outline 
- Selecting Contexts for Each Text (Descriptors) - For each keyword a set of preliminary contexts is extracted from the Internet, which is used as a context database.

- Ranking the Contexts - Each preliminary context is ranked according to the number of references it receives in the context database and the number of appearances it has in the text.

- Identifying the Current Contexts - The preliminary contexts that have significantly higher numbers of references and higher numbers of appearances are included in the current set of contexts.

- Uniting Synonymous Contexts - The current contexts are examined for synonyms and synonymous contexts are united.

\subsection{Collecting Data}

Each text is decomposed into single words, when words are letter strings separated by spaces, and all punctuation is removed from the text. Then the words are checked according to a set of dictionaries. The first dictionary is a "Stop List", consisting of words that do not add to the understanding of the context, such as I, me, in, are, the. All words that appear in this dictionary are ignored. The next step uses a set of dictionaries according to fields of knowledge to sieve the words that are not related to the specific field of knowledge. If the word appears in the field of knowledge dictionary, then it is added to the list of keywords that are searched in the context database, otherwise it is ignored. This process continues for each word in the text. After each text passes through this module, the algorithm sends a list of words to be checked for a possible set of contexts. Checking against a dictionary can be skipped if the field of knowledge is unknown, but skipping this step may sometimes lead to less accurate results. The difficulty does not lie in finding the possible keywords since the algorithm can always use the whole input corpus.

\subsection{Selecting Contexts for Each Text (Descriptors)}

The selection of the current context is based on a search through the database for all relevant documents according to keywords and on the clustering of the results into possible contexts. Once a list of keywords exists, each keyword is searched in the context database - the Internet - and a set of contexts is extracted. This creates a list of preliminary contexts for each keyword. The contexts in this work were represented by words or sets of words, which can be viewed as meta data created for each set of Internet web pages. Other descriptors can include images appearing on the Internet. The Internet can then be seen as a vast set of descriptors that represent different possible contexts, each associated with its respective web page. The full list of preliminary contexts for all the keywords includes all the possible contexts for this current text.

Any search engine can be used and any Term Frequency / Inverse Document Frequency [5] method for clustering can be implemented. The current application of the algorithm uses the concise all pairs profiling (CAPP) clustering method. [13], as it is applied in the Vivisimo search engine.

Using the Internet as a context database instead of a precalculated frequencies base has several advantages. The use of the Internet does not require the constant 
updating and maintenance of a database. The precalculated frequencies base requires the user to work in a limited predefined knowledge domain. The Internet can serve as an unlimited knowledge domain that is being continuously updated. This step results in a long list of preliminary contexts, many of which are irrelevant to the context. The purpose of the following steps is to minimize the list and identify most relevant contexts to the situation.

\subsection{Ranking the Contexts}

New preliminary contexts can now be created according to textual sub-strings of existing preliminary contexts. This step sums the number of documents referring to the preliminary contexts. Multiple reference pages from similar web sources are counted as one instance. Each document usually refers to multiple contexts, consequently creating a long list of preliminary contexts. The last step involves ranking the set of preliminary contexts according to both the number of references from the documents and the number of appearances in the text. This step maps all the preliminary contexts to a two dimensional graph, allowing the contexts that receive very high ranking in both characterizations to be located, as in Figure 2. After each session of ranking, the list is used for two purposes - resetting the set of preliminary contexts and identifying the current context. The current list of contexts joins the new preliminary contexts arriving from the continuously streaming text. The lists are united and the ranking process is repeated. In parallel to the repetition of the ranking algorithm, the set of ranked preliminary contexts is forwarded to the next module to determine the current contexts.

\subsection{Identifying the Current Contexts}

The output of the ranking stage is the current context or a set of highest ranking contexts that differ essentially by rank. The algorithm then returns to the first step to collect more texts and feed them again to the database. The set of preliminary contexts that has the top number of references, both in number of Internet pages and in number of appearances in all of the texts, is defined as the highest ranking and is identified to be the current contexts.

The current contexts received from the previous stage can be depicted on a graph according to number of appearances and number of references as in Figure 2. The algorithm for detecting the current contexts includes the following steps:

1. Organize the list of preliminary contexts in descending order according to number of references appearing in the Internet - the Set of Documents.

2. Find the difference between each value of the number of references and its nearest lower value neighbor, defined as Current References Difference Value (CRDV).

3. Find the difference between each value of the number of appearances and its nearest lower value neighbor, defined as Current Appearance Difference Value (CADV).

4. Weight the number of appearances in the text and the number of references in the Internet according to the following formula:

MVR = Maximum Value of References

MVA = Maximum Value of Appearances

WeightedValue $=\sqrt{\left(\frac{2 * C A D V * M V R}{3 * M V A}\right)^{2}+(C R D V)^{2}}$ 
The Weighted Value can be viewed as the weighted distance to the origin. However, the index of number of references is on a much larger scale than the index of the number of appearances and therefore it is not possible to retain the original proportions and it is necessary to re-scale the indices. The value is calculated multiplying by the maximum number of references and dividing by the number of maximum number of appearances. A constant of $2 / 3$ was found by experiment to be appropriate for the re-adjustment of the figures.

5. Find the maximum value of the Weighted Value. If the maximum Weighted Value is the first value, then continue to the next one, since frequently the first value is too far from its neighbor.

6. Select all the contexts that appear before the maximum Weighted Value in the list that was organized in step 1 as the current contexts. Store current selected contexts.

7. Erase the selected contexts from the list and repeat steps 5 and 6 again.

The first cluster of contexts near the origin includes all the contexts that received low ranking both in number of appearances and in number of references. This group of contexts includes most of the contexts in the list. Since the contexts in this group received low ranking they are eliminated from the list. The remaining contexts are the current contexts. The process can continue until all the contexts in the list are covered and this will yield all the possible preliminary contexts. However, in most cases the best results were already achieved when steps 5 and 6 were performed two times. Further repetitions, which increase the number of results, were unnecessary. The ranking according to number of references and not according to the weighted value also improves the results. This indicates that the number of appearances of the context in the text has less value in the determination of the context than the number of references in the Internet. During the implementation of the algorithm, there was a problem that required special consideration. The contexts that received lower ranking than the top ranking contexts in the cluster but were not part of the cluster were kept. Namely, these are contexts that receive lower ranking in either the number of appearances or the number of references than the top ranking contexts, but not in both. Running the algorithm showed that these contexts are sometimes relevant and should be kept.

\subsection{Uniting Synonymous Contexts}

The current contexts are examined for synonyms using a thesaurus and synonymous contexts are united. Before this step, many of the contexts identified by the algorithm are similar in meaning. This step enables the algorithm to identify the differing multiple contexts of the situation and thus facilitates the better description of the situation.

The current set of contexts is the output of the algorithm. However, since the algorithm is continuous, the contexts continue updating as long as new textual input continues to be accessed by the algorithm.

Example 1. The example presents the implementation of the algorithm on text taken from MSN chat and the results of the algorithm.

Jane: NO I DONT THINK SO IVE DONE ALOT OF SEARCHING THE NET FOR INFO

Jane: HAVE GATHERED ALOT 


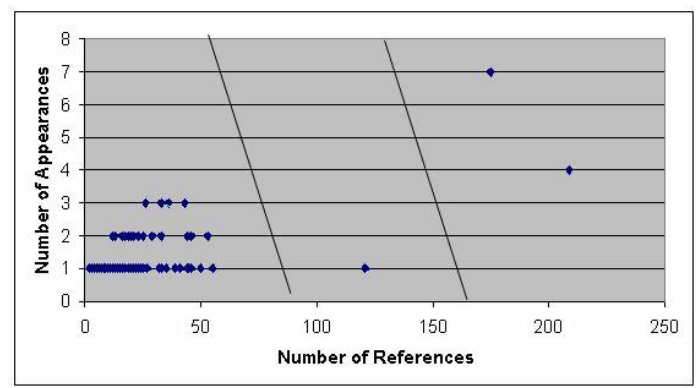

Fig. 2. Identifying the Current Contexts

Lisa: are you a diabetic pt.?

Jane: YES

Jane: TYPE 2

Jane: 1 YR

Jane: AND A BIT OVERWEIGHT BUT NOT OBESE

Lisa: ok. are you married?

Jane: YEP

Jane: 23 YRS

Lisa: do you have children?

Jane: HAPPILY MARRIED

Lisa: do u control your blood gloucose level

Jane: YES DIET AND MEDICINE

Jane: AND EXERCISE

Lisa: is there any complications like eye problems

Jane: I WEAR GLASSES

First the input is read one line at a time. Each word is separated by a space. Punctuation marks are eliminated. Each word is checked against the "Stop List" dictionary. In this case each word was checked in a predefined medical dictionary. The words that passed the previous stage serve as keywords. After each step (change of speaker) the keywords are sent to the search engine and clustered into a list of preliminary contexts. These steps are repeated 17 times, yielding 139 preliminary contexts that have at least two references in the Internet and are relevant to keywords that appeared at least once in the text.

The algorithm maps all the preliminary contexts to a two dimensional graph, allowing the contexts that receive very high ranking in both characterizations to be located, as in Figure 2.

The set of identified contexts includes: Diabetes $(209,4)$, Eye $(175,7)$, and Diabetes Type $2(121,1)$. The value in the parentheses includes the number of references and the number of appearances respectively. Figure 2 shows the weighted value calculated for each context. The differences show that the maximum weighted value is after Eye (69.66), resulting in two contexts: Diabetes and Eye. The second maximum weighted value is after Diabetes Type 2 (67.61), resulting in only one additional context, Diabetes Type 2. As a result the algorithm selected the first three contexts - Diabetes, Eye, and Diabetes Type 2 - as the current contexts. 
Example 2. The algorithm was run without any information about the knowledge domain and did not use any field of knowledge dictionary. The results of algorithm in the following example included the contexts of Hacker and Security. This shows that preliminary contexts can also be words that did not appear in the text itself but were a result of the clustering of the web pages, as in the top ranking context of Security.

Fox-Fire1: i want to ask some question about hacking

Fox-Fire1: any body help me

simply-crazy: h word is bad

mad-for-computers: what ques about haciking

mad-for-computers: hacking

mad-for-computers: i love hacking

Fox-Fire1: some body hack my id

Demon11: !illegal

Obis-Shadow: Please note: Owner, hosts and participants of this room we don't offer any help with illegal activity. This includes p2p software.(such as Kazaa and Imesh). any discussion of such activities will result in banishment from chat.

Fox-Fire1: and i want to get it back

Fox-Fire1: ohh

Fox-Fire1: hi

Fox-Fire1: what happened

mad-for-computers: nothing

mad-for-computers: there was room named $h$ but it is gone

Fox-Fire1: o k tell me how i can get back my id

Demon11 and Obis-Shadow are software bots that monitor the chat. Eventually the participant Fox-Fire1 was banished from the chat as a result of raising an illegal discussion topic - hacking, although the participants were discussing security measures against hacking.

\section{Analysis}

The context recognition algorithm was evaluated using computer-related Internet chats acquired from MSN chats. The chats included a few participants and were observed over time. Parts of the chats that dealt with topics concerning computers were copied to files. From these chats sets of files were randomly selected to be analyzed by the algorithm. These files were fed as input to the context algorithm. The results were compared with the results given by computer-literate subjects.

The subjects who answered the survey were graduate students with at least basic knowledge of computer terminology. A total of 20 subjects participated in the survey. The participants received a set of 3 chats. This allowed two groups of participants to be formed, each group with a different set of chats. Each chat had at least 9 replies. The maximum number of replies per chat was 11 . An average of 10 subjects determined the list of contexts for each chat. A total of six chats in computer related topics were analyzed in this way. The subjects were presented with the above chats and were asked to provide a list of contexts for the chats. The subjects were told that the text was obtained from Internet chats and was presented to them as is, including spelling mistakes and Internet acronyms. The subjects were asked to write in their own words what they felt were the contexts. These words were not selected from a list. The words were counted to determine the number 
of times they appeared among all the subjects. Each word was counted once for each subject who mentioned the context. The context was ranked in descending order according to the number of times that the subjects mentioned the context. The list of best-ranked contexts was compared with the results yielded by the context recognition algorithm. The other contexts mentioned more than once by the subjects were also compared to check the sensitivity of the algorithm.

Table 1 summarizes the comparison of the results of the algorithm and the results of the subjects. The table also displays the average results of the contexts recognition algorithm for all six cases examined. The second and third sets of contexts, which are contexts mentioned by the subjects but not selected by a majority of the people or only selected by one or two subjects, respectively, are also compared with the second and third reiterations of the algorithm to check the sensitivity of the algorithm.

Table 1. Ranked Contexts (RC) of the Algorithm

\begin{tabular}{|l|ccccccc|}
\hline Contexts Recognition & Chat 1 & Chat 2 & Chat 3 & Chat 4 & Chat 5 & Chat 6 Average \\
\hline Top RC & $100 \%$ & $100 \%$ & $100 \%$ & $100 \%$ & $100 \%$ & $100 \%$ & $100 \%$ \\
Top and II RC & $100 \%$ & $84.46 \%$ & $65.22 \%$ & $57.15 \%$ & $75 \%$ & $100 \%$ & $80.31 \%$ \\
Top, II, and III RC & $86.67 \%$ & $81.25 \%$ & $57.15 \%$ & $40 \%$ & $76.92 \%$ & $75 \%$ & $69.50 \%$ \\
\hline
\end{tabular}

The top ranking contexts mentioned by the subjects were identified as contexts by the algorithm. In most of the cases the contexts that were ranked among the highest by the subjects were also ranked among the highest by the algorithm. Some of the other contexts generated by the algorithm were not selected by the subjects. In addition, a few of the lower ranking contexts mentioned by the subjects were missed by the algorithm. Table 1 shows that for the top ranking contexts the algorithm yields very high results. As more contexts that received lower ranking by the subjects are added, the results of the algorithm degenerate. The algorithm needs improvement in deducting better results in the second and third ranking sets of contexts.

The significance of the results was analyzed using the identical populations test. The test for homogeneity is designed to test the null hypothesis that two or more random samples are drawn from the same population or from different populations, according to some criterion of classification applied to the samples. The Chi-square Pearson Test for Association is a test of statistical significance. The results of the identical populations test comparing the groups containing the algorithm as a subject with the original group consisting only of human subjects showed that they were almost identical populations. In other words, if the computer is part of the group, the context will remain identical. Hence, there is no significant difference in the determination of context between the algorithm and the human subjects. Table 2 displays the identical population test results for each of the six chats.

Currently the algorithm is being analyzed in the fields of medical case studies and eGovernment services. In the field of medical case studies, the context recognition algorithm is used to extract information from actual medical cases. The goal is to examine a method for encapsulating a patient's medical history and current situation into keywords for the physician to assist his analysis. In the field 
Table 2. Identical Populations Test

\begin{tabular}{|l|cc|}
\hline Chat & $\chi^{2}$ & P-Value \\
\hline 1 & 0.065199 & 1 \\
2 & 0.568693 & 0.999 \\
3 & 0.187391 & 1 \\
4 & 0.256795 & 1 \\
5 & 0.133712 & 1 \\
6 & 0.273300 & 0.998 \\
\hline
\end{tabular}

of eGovernment services, the algorithm is currently being examined in TERREGOV and QUALEG, European IST projects. TERREGOV aims at providing territorial governments with flexible and interoperable tools to support the change towards eGovernment services. The purpose is to identify the contexts of documents to enable the revision of ontologies for optimization of indexing and search of documents. QUALEG aims at providing local government an effective tool for bi-directional communication with citizens. Contexts are used to specify citizen input and then provide services - routing emails to departments, opinion analysis on topics at the forefront of public debates, and identification of new topics on the public agenda.

\section{Conclusion}

Every situation can be characterized by multiple contexts that describe its different aspects and that are necessary for the complete understanding of the different perspectives of the situation. The main idea of the research was to use the Internet as a context database to identify the multiple contexts of the given situation. The Internet is a source of information that is constantly increasing and being updated. The use of the Internet as a database for context recognition therefore gives a context recognition model immediate access to a nearly infinite amount of data in a multiplicity of fields. Hence, the necessity of creating a database for the determination of the context is eliminated.

Furthermore, the situations for which the context is sought can be independent of the Internet; the Internet is merely the database in which the algorithm searches for the context. Thus, for example, the context of a conversation between people can be found through the use of the Internet - the algorithm is a tool that allows the computer to determine the context by using the Internet as a database and then to pass this context back into the real world. The Internet is one possible source of data, but the algorithm holds also for a more restricted database. Intranet data, internally generated textual information about the organization that is stored, can also be used.

The complexity of the algorithm is directly dependent on the size of the input description of a given situation and thus online implementation is feasible. Currently, it is being implemented in two online eGovernment applications.

Initial tests show that the algorithm also achieves good contexts recognition results without the use of a field of knowledge dictionary, which represents specialized knowledge. Thus the algorithm can be used in diverse areas without predefined knowledge of the field. 
The Internet includes many different representations of data, such as text, image, and sound. Therefore, future directions of research include implementing the algorithm to extract contexts in alternative formats of representation. Other directions of research include mapping multiple contexts to ontologies, since contexts and ontologies are complementary disciplines of modeling views.

\section{Acknowledgments}

The work of Segev was partially supported by two European Commission $6^{\text {th }}$ Framework IST projects, TerreGov (http://www.terregov.eupm.net) and QUALEG (http://www.qualeg.eupm.net), and the Fund for the Promotion of Research at the Technion.

\section{References}

1. In AAAI'99 (1999) Workshop on Reasoning in Context for AI Applications, July 191999.

2. S. Dumais and H. Chen. Hierarchical classification of web content. In Proceedings of SIGIR, 23rd ACM International Conference on Research and Development in Information Retrieval, 2000.

3. L. Erman, F. Hayes-Roth, V. Lesser, and D. R. Reddy. The Hearsay II speech understanding system: Integrating knowledge to resolve uncertainty. Computing Surveys, 12(2):213-253, 1980.

4. A. Gal, G. Modica, H.M. Jamil, and A. Eyal. Automatic ontology matching using application semantics. AI Magazine, 26(1), 2005.

5. S. Gerard. Automatic Text Processing: The Transformation, Analysis, and Retrieval of Information by a Computer. Addison-Wesley Publishing Company, Inc., 1989.

6. S. Vadrevu H. Davulcu and S. Nagarajan. Ontominer: Bootstrapping and populating ontologies from domain specific websites. In Proceedings of the First International Workshop on Semantic Web and Databases, 2003.

7. V. Kashyap, C. Ramakrishnan, C. Thomas, and A. Sheth. Taxaminer: An experimentation framework for automated taxonomy bootstrapping. International Journal of Web and Grid Services, Special Issue on Semantic Web and Mining Reasoning, September 2005. to appear.

8. A. Maedche and S. Staab. Ontology learning for the semantic web. IEEE Intelligent Systems, 16, 2001.

9. J. McCarthy. Generality in artificial intelligence. Communication of ACM, 1987.

10. J. McCarthy. Notes on formalizing context. In Proceedings of the Thirteenth International Joint Conference on Artificial Intelligence, 1993.

11. R. M. Turner. Model of explicit context representation and use for intelligent agents. In 1999 International and Interdisciplinary Conference on Modeling and Using Context (CONTEXT-99), 1999.

12. D. P. Turney. Mining the web for lexical knowledge to improve keyphrase extraction: Learning from labeled and unlabeled data. ERB-1096 NRC \#44947, National Research Council, Institute for Information Technology, 2002.

13. R. E. Valdes-Perez and F. Pereira. Concise, intelligible, and approximate profiling of multiple classes. International Journal of Human-Computer Studies, pages 411-436, 2000. 